\title{
PRINCIPIOS BÁSICOS Y FUNDAMENTOS DE LA INCLUSIÓN EDUCATIVA. EL CASO DE LA UNIVERSIDAD AUTÓNOMA DEL ESTADO DE HIDALGO, MÉXICO
}

\author{
Maricela Zúñiga Rodríguez \\ Profesora investigadora del Área Académica de Ciencias de la Educación \\ del Instituto de Ciencias Sociales y Humanidades, Universidad Autónoma del Estado de Hidalgo, México. \\ maricela_zuniga@uaeh.edu.mx
}

Recepción Artículo: 07 septiembre 2021 Admisión Evaluación: 07 septiembre 2021 Informe Evaluador 1: 14 septiembre 2021 Informe Evaluador 2: 15 septiembre 2021 Aprobación Publicación: 18 septiembre 2021

\section{RESUMEN}

Para la UNESCO (2013), la infancia que tiene el derecho prioritario a una educación inclusiva son: Ios niños migrantes; Ios niños de la calle; los niños obreros; Ios niños soldados; Ios niños con discapacidad; los pueblos indígenas y las poblaciones rurales. Si bien la política de educación inclusiva de la UNESCO está enfocada a la infancia y al sistema educativo básico. Es necesario que esta cobertura alcance a la población de adolescentes y jóvenes que cursan el sistema del nivel medio y superior. Las instituciones que los atienden están enfrentando el desafío que implica la educación inclusiva sin el respaldo de políticas públicas. Ante este desafío la presente comunicación tiene como objetivo identificar los principios y fundamentos en la implementación de la educación inclusiva en la educación superior a través del estudio del caso en una universidad pública en el estado de Hidalgo, México, el escrito se integra por los antecedentes de la educación inclusiva en el nivel superior, el objetivo de estudio, la metodología y técnicas empleadas a través de un estudio de caso, los resultados, las conclusiones obtenidas y las referencias bibliográficas consultadas.

Palabras clave: educación inclusiva; educación superior; principios; fundamentos

\section{ABSTRACT \\ Basic principles and foundations of educational inclusion. The case of the Autonomous University of the State of Hidalgo, México. For UNESCO (2013), the children that have the priority right to inclusive education are: migrant children; street children; working children; child soldiers; children with dis- abilities; indigenous peoples and rural populations. While UNESCO's inclusive education policy is focused on childhood and the basic education system. It is necessary for this coverage to reach the population of adolescents and young people who attend the middle and higher level system. The institutions that serve them are facing the challenge of inclusive education without the support of public policies. Faced with this challenge, this communi- cation aims to identify the principles and foundations in the implementation of inclusive education in higher edu-}




\section{PRINCIPIOS BÁSICOS Y FUNDAMENTOS DE LA INCLUSIÓN EDUCATIVA. EL CASO DE LA UNIVERSIDAD AUTÓNOMA DEL ESTADO DE HIDALGO, MÉXICO}

cation through the case study in a public university in the state of Hidalgo, Mexico, the writing is integrated by the background of inclusive education at the higher level, the study objective, the methodology and techniques used through a case study, the results, the conclusions obtained and the bibliographic references consulted.

Keywords: inclusive education; higher education; beginning; fundamentals

\section{ANTECEDENTES}

El enfoque de la Educación Inclusiva ha buscado impactar en los sistemas educativos de todo el mundo con el fin de dar respuesta a la diversidad de los estudiantes. Al respecto la UNESCO considera que se debe prestar especial atención a los grupos marginados y vulnerables -personas y grupos que no pueden ejercer su derecho a la educación- para procurar desarrollar todo el potencial de cada persona. Es así como considera que "la educación inclusiva y de calidad se basa en el derecho de todos los alumnos a recibir una educación de calidad que satisfaga sus necesidades básicas de aprendizaje y enriquezca sus vidas" (UNESCO, 2013).

La UNESCO en su lucha contra la exclusión y con la labor de terminar con cualquier tipo de discriminación y fomentar la cohesión social declara que la educación inclusiva y de calidad se basa en el derecho de todos los alumnos a recibir una educación que satisfaga sus necesidades básicas de aprendizaje y enriquezca sus vidas ya que al prestar especial atención a los grupos marginados y vulnerables, la educación integradora y de calidad procura desarrollar todo el potencial de cada persona (UNESCO, 2013).

En este sentido, universidades públicas y privadas en distintos países han sido de gran ejemplo mostrando el impacto de sus programas o planes de inclusión educativa en la formación de profesionistas. En México, universidades públicas y privadas se han dado a la tarea de proporcionar educación a personas en situación de vulnerabilidad por ejemplo: la Universidad Autónoma de México, Universidad Veracruzana, Universidad Lasalle, entre otras. Algunas de ellas con programas específicos para este fin y otras sin ningún tipo de respaldo institucional pero con gran mérito en la práctica. Las prácticas educativas habituales realizadas por las universidades públicas de México, se transforman ante esta gran laborante la inclusión de personas en situación de vulnerabilidad.

La Educación inclusiva debe verse como un eje de transformación de las universidades, y armonizar la reducción de las desigualdades que impiden efectivizar el derecho a la educación y el aprendizaje comprender la diversidad de expectativas y de necesidades de todos los estudiantes como oportunidades para ampliar y democratizar los aprendizajes.

En 2017 la UNESCO en el XIV Congreso Internacional de Educación Inclusiva y XXXIV Jornadas de Universidades y Educación Inclusiva en Oviedo, España, publica las nuevas Directrices de política sobre la inclusión y la equidad en la educación con el propósito de apoyar a los países para asegurar que inclusión y equidad sean transversales a los sistemas educativos.

Las directrices proponen tomar en cuenta los siguientes fundamentos:

1. Las políticas educativas hacia la inclusión educativa se realicen en todos los niveles educativos de los sistemas educativos.

2. Reconocer que el cambio educativo es técnicamente sencillo, pero social y políticamente complejo.

3. La claridad de los objetivos es necesaria para movilizar un amplio apoyo a las poblaciones en situación de vulnerabilidad.

4. Registrar la evidencia como catalizador de un cambio exitoso.

Estas directrices están pensadas como inclusión y equidad definidas como:

Inclusión: que entraña superar los obstáculos que limitan la presencia, participación, el bienestar y los logros de todos los alumnos y todas las alumnas.

Equidad: que consiste en asegurar que exista una preocupación por la justicia, de manera que la educación de todos los alumnos y alumnas se considere de igual importancia, (UNESCO, 2017).

Repensar los propósitos educativos y la concepción de una universidad inclusiva incluyente encara los siguientes desafíos: 
1. El fortalecimiento de una visión holística, humanista y transformadora de la educación, así como un enfoque del sistema educativo unitario e integrado.

2. La educación inclusiva entendida como un principio transversal de las políticas educativas y como una estrategia institucional, docente, curricular y pedagógica, que efectiviza el derecho de cada estudiante a tener una oportunidad real de educarse y de aprender.

3. Un entendimiento afinado acerca de las expectativas y necesidades de aprendizaje de los alumnos, reconociendo la naturaleza colectiva de los aprendizajes y la singularidad de la respuesta educativa facilitada a cada alumno/alumna así como recreando relaciones de confianza entre docentes y alumnos.

4. La toma de conciencia acerca que las respuestas genuinas al logro progresivo de la inclusión no parezcan estar pautadas principalmente por la adherencia a un paradigma ideológico en particular sino por la búsqueda de respuestas potentes frente al para qué, el qué, cómo, en dónde educar y aprender, (UNESC0, 2017).

En el artículo "Las instituciones de educación superior y los estudiantes con discapacidad en México", desarrollado a partir de una investigación sobre inclusión de estudiantes con discapacidad realiza un análisis detallado del panorama nacional sobre inclusión educativa en instituciones de educación superior en México, identificando que de 53 universidades, solo 12 tienen acciones enfocadas al apoyo de estudiantes con discapacidad, siendo la Universidad Autónoma del Estado de Hidalgo, considerada uno de los espacios con mayores estrategias de inclusión (Cruz \& Casillas, 2017), razón que motivo el presente estudio.

\section{OBJETIVO DE LA INVESTIGACIÓN}

Identificar los principios y fundamentos en la implementación de la educación inclusiva en la educación superior a través del estudio del caso de una universidad pública en el estado de Hidalgo, México.

\section{MUESTRA Y/O PARTICIPANTES}

El estudio surge a raíz de que esta universidad pública obtuviera cuatro estrellas en lo general por parte del sistema de rating QS Stars que analiza a las universidades conforme a una auditoria donde se refleja las fortalezas y áreas de oportunidad de cada institución de educación superior. La Unidad de inteligencia QS Stars, a través de una recopilación de datos rigurosa y un análisis de métricas, la máxima casa de estudios de la entidad fue calificada con cinco estrellas en las categorías de Docencia, Empleabilidad, Desarrollo Académico, Instalaciones, Inclusión, así como Arte y Cultura.

QS Stars valora a cada una de las casas de estudios por separado, según el desempeño de sus diferentes áreas. Se le juzga conforme al estándar que establece esta organización y da un resultado particular obteniendo, es decir, el rating no compara universidades entre sí, a diferencia de lo que hace el ranking, que da un posicionamiento general en un listado mundial. Por tal motivo se decidió estudiar cómo se llevan a cabo las directrices y fundamentos para llevar a cabo la Inclusión Educativa.

\section{METODOLOGÍA Y/O INSTRUMENTOS UTILIZADOS}

El conjunto de estrategias metodológicas que guían el trayecto del presente estudio, tiene sus bases en el diseño del estudio de caso, elegido en función de la naturaleza de la problemática y las características de la modalidad educativa y del contexto considerando las cualidades y los alcances.

Con antecedentes disciplinares en la psicología, la sociología, la antropología y la historia por mencionar algunas ciencias, el estudio de caso ha evolucionado para validarse como metodología pertinente que da cuenta de situaciones problemáticas singulares, las cuales pueden centrarse en torno a una persona, un aula, una institución, una política o un programa, (Simons, 2011).

El estudio de caso desde el enfoque cualitativo, Según Stake, (1995, citado en Simons, 2011) "... es el estudio de la particularidad y la complejidad de un caso por el que se llega a comprender su actividad en circunstancias que son importantes" (p. 40). Esta definición, deja ver la importancia de justificar la elección del contexto de investigación desde su relevancia, los sujetos y las condiciones sociales y culturales que lo configuran. 
Es importante mencionar que, diversos autores han establecido tipologías de estudio de caso, según el interés, el énfasis en el tema o problema o el número de casos seleccionados para investigar. La tipología que aquí se presenta, tiene sus bases en Stake (1998) quien distingue el caso intrínseco, cuando es seleccionado por su naturaleza y éste no depende de su relación con otro; el instrumental, cuando el caso se escoge para estudiar un tema o para entender o explicar una cosa y el colectivo, cuando se estudian varios casos para hacer una interpretación colectiva del tema de estudio. Desde esta clasificación, es conveniente definir que, el tipo de estudio de caso seleccionado, es el instrumental. Una característica que se asocia con esta tipología, es la necesidad de conocer de manera profunda sobre ese caso en particular, donde la elección viene dada e interesa por una problemática identificada en el contexto seleccionado, (Stake, 1998).

En la recolección de evidencia empírica, las técnicas e instrumentos de investigación ocupan un lugar indispensable, pues a través de éstas, se orienta la recuperación de información necesaria para profundizar en el conocimiento del objeto de interés. Para el presente estudio se empleó la técnica de análisis de documentos que consiste en recoger reflexiones sobre el contenido de documentos de interés acerca del objeto con la finalidad de obtener información importante sobre el problema a través de la revisión de documentos empleando como instrumentos fichas analíticas.

\section{RESULTADOS ALCANZADOS}

\section{Descripción del caso}

La Universidad Autónoma del Estado de Hidalgo (UAEH) es la más grande universidad pública en el estado de Hidalgo, México. Como Institución Pública tiene un compromiso con su entorno y con la familia humana de la sociedad global. Ofrece una educación con calidad y excelencia como derecho fundamental y bien público que pertenece a todas las personas. La oferta educativa de la UAEH cubre todas las áreas del conocimiento, en los niveles medio superior y superior, en las modalidades presencial y no presencial. Se integra por 121 Programas Educativos, de los cuales, uno corresponde a bachillerato; 62, a licenciatura; 14, a especialidad; 29, a maestría y 15 , a doctorado.

Se atienden una matrícula total de 64490 alumnos.

Sus principios son:

I.- Gobernabilidad: es la universidad más antigua del estado de Hidalgo con 151 años de tradición y 59 años de autonomía universitaria.

II.- Calidad: En la UAEH reciben formación integral con valores y cuentan con una amplia oferta de actividades culturales y deportivas. El Modelo Educativo está centrado en el alumnado. En la UAEH la equidad de género y el respeto a los derechos humanos es una norma permanente del quehacer institucional.

Tiene como Misión:

"Formar capital humano de alta calidad, de acuerdo con las necesidades de la sociedad global, propiciando la incorporación exitosa de sus egresados al trabajo productivo en el ámbito de su competencia; generar investigación de alta competitividad en beneficio de la sociedad, contribuyendo a la solución de problemas estructurales relacionados con la sostenibilidad y desarrollo de los sectores social, productivo y público; crear, preservar y difundir la cultura en beneficio de todos los sectores de la población, fomentando la solidaridad social y la preservación del patrimonio multicultural, étnico y natural; articular las funciones sustantivas de la universidad con el entorno mundial; fomentar la legalidad, transparencia y protección de los derechos humanos; planear, operar y gestionar en el ámbito académico y administrativo bajo el proceso permanente de la evaluación", (UAEH, 2021).

Su visión es:

Un aspecto que la institución ha consolidado desde hace más de tres décadas, es el trabajo basado en la planeación estratégica y el respeto irrestricto a la normatividad. El sustento de la Universidad tiene su origen en el 
Proyecto Integral de Transformación Académica, (PITA). Su visión se proyecta para los siguientes 20 años en ser una universidad visible internacionalmente y aceptada por sus resultados en materia de calidad académica y administrativa, como una institución educativa de calidad y prestigio, como una universidad global, reconocida por sus indicadores de excelencia y visible en los sistemas de medición internacional de la calidad académica como una de las mejores del mundo, (UAEH, 2021).

\section{Directrices y fundamentos identificados en la UAEH ante la Inclusión Educativa}

Dentro de la Universidad Autónoma del Estado de Hidalgo, se identificaron las políticas y documentos normativos que impactan en la inclusión de estudiantes en situación de vulnerabilidad, así como una investigación realizada sobre inclusión educativa en la UAEH.

El primer documento analizado es el Modelo Educativo (2015), documento rector que establece los principios y fundamentos bajo los que se establece la razón de ser de la UAEH en los ámbitos pedagógico, sociológico, jurídico, político y operativo. Dentro de las características que la educación que ofrece, se rescatan dos puntos fundamentales, el primero es Educar para la Igualdad que menciona lo siguiente:

"Propiciar la valoración y respeto por la interculturalidad y garantiza la igualdad de oportunidades por género, edad y condición social...; creemos que debe ser accesible para todos a lo largo de toda la vida, colocando en el eje de todas las acciones universitarias a los estudiantes a fin de que puedan integrarse plenamente en el mercado laboral; procurando establecer las medidas necesarias para asegurar la coordinación y cooperación entre los diversos sectores y dentro de cada uno de ellos, para transformarse y provocar el cambio, al atender las necesidades sociales y fomentar la solidaridad y la igualdad" (UAEH,2015, p. 45).

El segundo es Educar de forma Integra/ donde establece que:

"Para desarrollar la personalidad del ser humano de forma equilibrada, considerándolo como un ser complejo (biopsicosocial), por lo que cada parte que lo constituye crece y evoluciona armoniosa y proporcionalmente para alcanzar su plenitud, considerando las potencialidades del individuo para desarrollar sus capacidades. El objetivo de la educación integral es desarrollar habilidades, capacidades, valores, actitudes y aptitudes intra e interpersonales que les permitan a los actores educativos interactuar en diversas situaciones" (UAEH, 2015, p. 45).

Si bien no existe una mención específica de los estudiantes en situación de vulnerabilidad, el modelo establece como característica la igualdad y además la formación integral que permita el desarrollo de todos los ámbitos del ser humano, con esto abre la posibilidad de inserción de la diversidad vulnerable de los estudiantes.

El siguiente documento es el Modelo Curricular Integral, (UAEH, 2009, p. 16) que se desprende de los planteamientos realizados en el Modelo Educativo pero que establece la operatividad en un ámbito práctico. Se desarrolla dentro de la dimensión social que "el currículo tendrá como base, la formación integral del profesional que responda a las exigencias del desarrollo social, científico y tecnológico de su localidad, de su nación; en forma tal, que en su inserción al mercado laboral sea capaz de producir transformaciones institucionales y disciplinares".

Dentro de los ejes transversales descritos en el documento se encuentra la Educación para la equidad, que establece que "toda persona tiene derecho a la educación y que el acceso a los estudios superiores será igual para todos, en función de los méritos respectivos... y hacer accesible a todos, en condiciones de igualdad total y según la capacidad de cada uno, la enseñanza superior....", (UAEH, 2009, p. 16).

También hace mención que los sistemas de educación superior deben: aumentar su capacidad para vivir en medio de la incertidumbre, para transformarse y provocar el cambio, para atender las necesidades sociales y fomentar la solidaridad y la equidad; preservar y ejercer el rigor y la originalidad científicos, con espíritu imparcial por ser un requisito previo decisivo para alcanzar y mantener un nivel indispensable de calidad; y colocar a los estudiantes en el primer plano de sus preocupaciones en la perspectiva de una educación a lo largo de toda la vida, a fin de que se puedan integrar plenamente en la sociedad. A su vez reitera el eje de la Educación Integral del Modelo Educativo como eje transversal dentro de todos los Programas Educativos que imparte la UAEH. 


\section{PRINCIPIOS BÁSICOS Y FUNDAMENTOS DE LA INCLUSIÓN EDUCATIVA. EL CASO DE LA UNIVERSIDAD AUTÓNOMA DEL ESTADO DE HIDALGO, MÉXICO}

Un tercer documento analizado fue el Plan de Desarrollo Institucional 2018-2023. Actualización 2021-2023, que establece la necesidad de llevar a cabo las directrices propuestas por la UNESCO en específico:

"La educación inclusiva entendida como un principio transversal de las políticas educativas y como una estrategia institucional, docente, curricular y pedagógica, que efectiviza el derecho de cada estudiante a tener una oportunidad real de educarse y de aprender". (UAEH, 2021, P. 27).

Establece el derecho universitario de sensibilizar a los universitarios en materia de derechos universitarios, flexibilidad, inclusión, equidad y atención a grupos vulnerables establecido de la siguiente forma:

"4.7 Inclusión social 4.7.1 Impulsar y ejecutar apoyos para la formación integral e inclusión social de estudiantes que provengan de localidades de alta y muy alta marginación, comunidades indígenas, estudiantes con discapacidad motriz y/o sensorial y estudiantes con vulnerabilidad académica, con la finalidad de facilitar su ingreso, permanencia y egreso" (UAEH, 2021, P. 87).

Un cuarto documento complementario analizado fue la tesis titulada "Experiencias educativas de sujetos en posición de discapacidad en el contexto de la Universidad Autónoma del Estado de Hidalgo", es un trabajo de carácter doctoral que presenta un abordaje metodológico bajo la orientación mixta y la utilización de estudio de caso, al analizar 5 casos de personas con discapacidad con edades de los 20 a los 56 años, estudiantes y egresados de la Universidad Autónoma del Estado de Hidalgo, (Martínez, 2016).

Del análisis documental se estableció en qué medida los principios y fundamentos se atienden a las Directrices establecidas por la UNESCO (2017) para que realmente se ejerzan el pleno derecho de los estudiantes en situación de vulnerabilidad, donde se identificaron diferentes barreras físicas, administrativas y académicas al interior de la UAEH.

\section{DISCUSIÓN}

¿En qué medida son las dificultades frente al desafío de lograr una educación con principios básicos y fundamentos de la inclusión educativa en una universidad pública? Es de esperar que en cada universidad en particular exista tensión cuando de inclusión y exclusión de grupos en situación de vulnerabilidad se trata; por ello se requiere de un conocimiento en profundidad de cada escenario en donde se pretendan aplicar los principios y fundamentos de la inclusión educativa.

La Declaración Mundial sobre Educación Superior en el Siglo XXI: visión y acción (1998) estableció como marco de acción prioritaria para el cambio y el desarrollo de la educación superior, que la verdadera igualdad de oportunidades se relaciona con las capacidades para actuar en la sociedad y por aumentar las posibilidades de las personas para la toma de decisiones; por lo que, es necesario avanzar hacia políticas sociales que sitúen a las personas en el centro de un proceso de desarrollo humano sostenible, garantizando beneficios mínimos para toda la población, que les permitan vivir con dignidad, valorando la diversidad y respetando los derechos de todas las personas, es indispensable y continúa siendo un reto.

Otra información encontrada que se presta a discusión es en el Capítulo VI donde se vinculan los referentes estatales, nacionales e internacionales en educación superior, se observa la alineación de los objetivos del Programa de Desarrollo Institucional 2018- 2023 (UAEH); con la agenda 2030 de la ONU se identifica que lo articula en el Objetivo 4.- Garantizar una educación inclusiva, equitativa y de calidad y promover oportunidades de aprendizaje durante toda la vida para todos. Con respecto al Programa Sectorial de Educación 2020-2024: se alinea al Objetivo: 1. Garantizar el derecho de la población en México a una educación equitativa, inclusiva, intercultural e integral, que tenga como eje principal el interés superior de las niñas, niños, adolescentes y jóvenes. Por otra parte con el Plan Estatal de Desarrollo 2016- 2022 actualizado se alinea con el apartado 3. Hidalgo con Bienestar 3.2. Educación de Calidad. Estos objetivos se resumen en el PDI de la UAEH en su apartado 11. Programa institucional de Evaluación para la mejora organizacional y de las personas. (PP.106-107).

Con base en el modelo educativo de la institución, la responsabilidad de equilibrar la mejora constante del quehacer de la universidad es consustancial al impulso de los procesos de innovación y desarrollo establecidos 
en los diferentes objetivos propuestos en el marco de los referentes estatales, nacionales e internacionales. Se observa la constante actualización de la planeación que busca, por una parte, dar respuesta a la problemática que se asume como reto y, por la otra, lograr un mayor beneficio social para su comunidad interna y su entorno, con miras a favorecer el desarrollo estatal y nacional, conforme lo determina la misión de la UAEH.

Las investigaciones sobre inclusión educativa, son actualmente tendencia en las agendas nacionales e internacionales. Dentro de los modelos educativos, se proponen directrices para la inclusión de personas en situación de discapacidad o quienes presentan alguna condición específica que incide en su manera de aprender. En educación superior, son escazas investigaciones al respecto. Si bien los organismos nacionales e internacionales, muestran a la inclusión educativa como una necesidad a cumplir son necesarios procesos de certificación en las instituciones de educación superior

El logro de una educación de calidad para todos requiere de políticas sensibles a esta diversidad de situaciones, a los factores que configuran la brecha entre la escuela y el contexto. El reconocimiento de la alineación de objetivos es el punto de partida para el desarrollo de estrategias pedagógicas e institucionales a la medida de cada escenario.

\section{CONCLUSIONES}

La UNESCO a través de su liderazgo intelectual busca transformar la visión de la educación inclusiva, a través de proponer Principios Básicos y Fundamentos de la Inclusión Educativa que si bien se adoptan en universidades públicas de México como es el caso analizado de la UAEH, como un medio para reforzar la educación para el desarrollo sostenible, a través de las oportunidades de aprendizaje a lo largo de toda la vida, el acceso equitativo a todos los niveles de la sociedad y a las oportunidades de aprendizaje, al interior de la UAEH como lo muestra el estudio de (Martínez, 2016) aún faltan acciones específicas como:

-Considerar el sustrato de socio-cultural, económico y político de la inclusión como parte de Programa de desarrollo institucional proyectado como parte de su Visión para el año 2030.

-La inclusión no es solamente acceso al espacio escolar sino el apoyo efectivo de los aprendizajes a través de la asequibilidad, como la disponibilidad de las políticas educativas para asegurar que en la institución existan recursos no solo físicos, sino personal, y programas educacionales de calidad, con la capacitación suficiente de acuerdo al contexto universitario.

Con respecto a las directrices se puede concluir que

La UAEH desde 2009 considera las políticas educativas hacia la inclusión educativa incorporándolo a su Modelo Educativo.

Se identifica que el cambio educativo inclusivo no ha sido sencillo y es políticamente complejo al ser un proceso gradual.

Existe claridad de los objetivos del Programa de Desarrollo Institucional de la UAEH, aunque hace falta movilizar un amplio apoyo administrativo, económico y curricular en beneficios directo de las poblaciones en situación de vulnerabilidad.

Se requiere de mayor investigación sobre el acceso, permanencia y egreso de estudiantes en situación de vulnerabilidad como catalizador de un cambio real y exitoso.

Si bien el modelo educativo de la UAEH considera políticas a nivel internacional delimita cuáles asume como propias, derivadas además de las tendencias mundiales de la educación superior. Los principios y fundamentos han sido guía de referencia a las demandas en el corto, mediano y largo plazo que se deben atender para lograr la consolidación y desarrollo institucional en términos de mejora de la calidad en ámbitos como la inclusión educativa, para ello se deberán repensar los propósitos educativos y la concepción de una universidad inclusiva e incluyente al encarar los fundamentos y principios propuestos por la UNESCO (2017). 


\section{REFERENCIAS BIBLIOGRÁFICAS}

Denzin, N. K. (2008). Los nuevos diálogos sobre paradigmas y la investigación cualitativa. Un compromiso en la relación universidad-sociedad. Reencuentro. Análisis de Problemas Universitarios (52), 63-76. https://www.redalyc.org/articulo.oa?id=34005206

Huberman, M. y Miles, M. (2000). Metodos para el manejo y el analisis de datos. En Denman, C. y Armando, H. (Comp.). Por los rincones. Antologia de metodos cualitativos en la investigacion social. El Colegio de Sonora.

Flick, U. (2015). El diseño de investigación cualitativa. Madrid. Morata.

Martínez, F. (2016). Experiencias educativas de sujetos en posición de discapacidad en el contexto de la Universidad Autónoma del Estado de Hidalgo. Tesis doctoral, Inédita. UAEH. México.

Simons, H. (2011). El estudio de caso: Teoría y Práctica. Morata. España.

Stake, R. E. (1998). Investigación con estudio de casos. Morata. España.

Strauss, A. y Corbin, J. (2002). Bases de la investigacion cualitativa. Tecnicas y procedimientos para desarrollar la teoria fundamentada. Universidad de Antioquia.

UNESCO (2008). Educación inclusiva: El Camino del futuro. Italia: UNESCO.

UNESCO. (2016). Conferencia Mundial sobre Necesidades Educativas Especiales: Acceso y Calidad. Salamanca, 1994. Recuperado de http://conadi.gob.gt/1/wp-content/uploads/2013/04/DeclaracionSalamanca.pdf

UNESCO. (2000). Educación para todos. Consultado el 15 de mayo DE 2016. En: http://www.unesco.org/new/es/our-priorities/education-for-all/

UNESCO (2017). Declaración Mundial sobre la Educación Superior en el Siglo XXI. Consultado el 5 de septiembre de 2017. www.unesco.org/education/educprog/wche/declaration_spa.htm

UNESCO (2017). XIV Congreso Internacional de Educación Inclusiva y las XXXIV Jornadas de Universidades y Educación Inclusiva sobre «Prácticas innovadoras inclusivas: retos y oportunidades. Consultado en https://revistadepedagogia.org/.../xiv-congreso-internacional-de-educacion-inclusiva-

UAEH.(2021). Plan de Desarrollo Institucional 2018-2023 ACTUALIZACIÓN 2021-2023. UAEH: México. En: https://www.uaeh.edu.mx/excelencia/PDI_21-23.pdf

UAEH (2021). Confirma UAEH su calidad con cuatro estrellas en rating QS. En: Consultado el 1 de agosto de 2021.Starshttps://www.uaeh.edu.mx/noticias/6352/

https://www.uaeh.edu.mx/modelo_educativo/docs/sin_modelo_educ_pag.pdf 